\title{
Tunable silicon photonics directional coupler driven by a transverse temperature gradient
}

\author{
Piero Orlandi, ${ }^{1,3, *}$ Francesco Morichetti, ${ }^{2}$ Michael John Strain, ${ }^{3}$ Marc Sorel, ${ }^{3}$ Andrea Melloni, ${ }^{2}$ and Paolo Bassi ${ }^{1}$ \\ ${ }^{1}$ Dipartimento di Ingegneria dell'Energia Elettrica e dell'Informazione, Università di Bologna, Viale Risorgimento 2, Bologna 40136, Italy \\ ${ }^{2}$ Dipartimento di Elettronica, Informazione e Bioingegneria, Politecnico di Milano, Via Ponzio 34/5, Milano 20133, Italy \\ ${ }^{3}$ School of Engineering, University of Glasgow, Glasgow G12 8LP, UK \\ *Corresponding author: piero.orlandi@unibo.it
}

Received December 26, 2012; revised February 6, 2013; accepted February 6, 2013;

posted February 11, 2013 (Doc. ID 182055); published March 11, 2013

\begin{abstract}
A compact directional coupler fabricated on a silicon photonic platform is presented, with a power-splitting ratio that can be tuned through a transverse temperature gradient induced by a laterally shifted integrated heater. The tuning mechanism exploits the thermally induced phase velocity mismatch between the coupled modes of the silicon waveguides. The positions of the integrated heater and the waveguide design are optimized to maximize the tuning range and to reduce electric power consumption. Asynchronous devices with an intrinsic phase mismatch are demonstrated to be more efficient, providing a tunable coupled power from 0.7 to 0.01 with $36 \mathrm{~mW}$ maximum power dissipation. (c) 2013 Optical Society of America

OCIS codes: $130.0130,160.6840$.
\end{abstract}

Reconfigurable photonic integrated circuits (PICs) capable of dynamically adapting their characteristics to the requirements of time-varying optical systems are attracting increasing interest in a variety of application fields, including on-chip networking [1] , optical communication systems [2] microwave photonics [3] and general purpose optical processing devices [4]]. Depending on the specific architecture and technological platform, reconfigurable PICs require a number of tunable elements, such as variable phase shifters [1-5], active gain/loss waveguide sections [4] and controllable power-splitting devices [3,5], to either modify the frequency domain response or optimize the circuit performance by counteracting fabrication tolerances [5].

Tunable integrated power-splitting devices have been deeply investigated since the early stages of integrated optics, including thermally or electro-optically actuated directional couplers (DCs) [] $]$, asymmetric waveguide junctions [?] and multimode interference (MMI) couplers [8]. In silicon photonics the most common approach to realize a tunable coupler exploits a thermally actuated balanced Mach-Zehnder interferometer (MZI) [3,5]. Although being simple to implement and widely tunable with a moderate electric power consumption, the MZI tunable coupler requires a pair of $3 \mathrm{~dB}$ splitters, thus resulting in a larger device and higher insertion loss.

In this Letter we propose a compact tunable directional coupler (TDC) consisting of a single silicon photonic DC driven by the transverse temperature gradient induced by a laterally shifted heater. The tuning mechanism is based on the thermally induced phase velocity mismatch between the coupled modes of the silicon waveguides. The positions of the heater and the DC cross section are optimized to maximize the tunability range, while reducing both footprint and electric power consumption.

Figure 1(a) shows a cross-sectional sketch of the proposed device. A $480 \mathrm{~nm} \times 220 \mathrm{~nm}$ silicon core waveguide on a $2 \mu \mathrm{m}$ buried oxide (BOX) undercladding and a $700 \mu \mathrm{m}$ bulk silicon substrate is employed. In the coupling section of the TDC, two straight waveguides are spaced by a gap distance, $g$, and are embedded in an oxide bi-layer formed by spun and baked hydrogen silsesquioxane (HSQ) and $\mathrm{SiO}_{2}$ [9], resulting in an overcladding thickness $H=900 \mathrm{~nm}$. A NiCr heater with a width $w_{h}=900 \mathrm{~nm}$ and height $t_{h}=50 \mathrm{~nm}$ is placed on top of the overcladding at a distance, $d$, from the center of one of the coupled waveguides. The average heater resistivity is $1.14 \Omega \mu \mathrm{m}$. As shown in the photograph of Fig. 1(b), the heater length was designed to be equal to the straight coupling section $L_{c}$ and low resistivity Ti-Au strips were used to bring the electric signal to the TDC.

The coupled power $K$ between the waveguides of the TDC is given by [10]

$$
K=\frac{|\kappa|^{2}}{S^{2}} \sin ^{2}\left(S L_{c}\right),
$$

where $S=\left(|\kappa|^{2}+\Delta \beta^{2} / 4\right)^{0.5}, \kappa$ is the field coupling coefficient, $\Delta \beta=2 \pi \Delta n / \lambda$ is the phase mismatch term, and $\lambda$ is the optical wavelength. Switching the heater on induces a transverse temperature gradient $\Delta T$ proportional
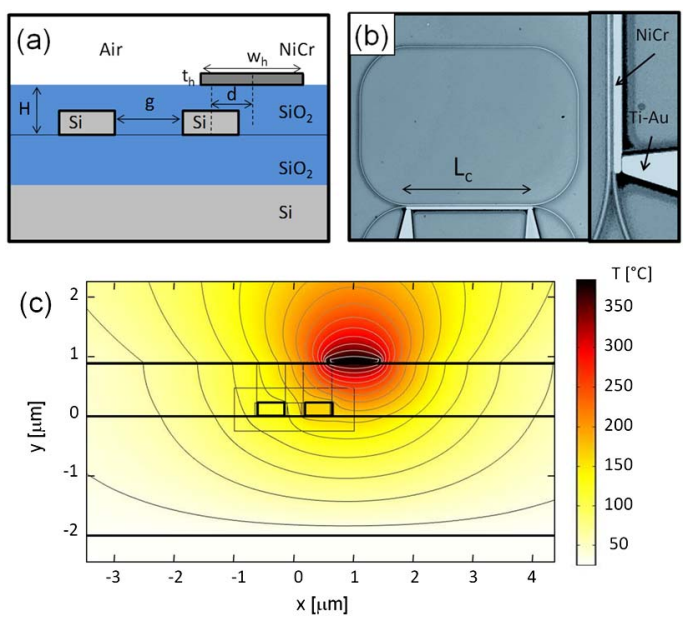

Fig. 1. (Color online) (a) Sketch of the TDC cross section. (b) Optical microscope top-view photograph of a silicon TDC embedded in an integrated RR all-pass filter. (c) 2D thermal FEM simulation of the silicon TDC cross section. 
to the dissipated electric power $P_{D}$ and provides an extra effective index difference $\Delta n_{t h}$ between the two waveguides that adds to the intrinsic one, $\Delta n$. Figure $1(\mathrm{c})$ shows a thermal simulation (done with COMSOL Multiphysics) of the TDC cross section for $P_{D}=34.5 \mathrm{~mW}$ and $L_{c}=74 \mu \mathrm{m}$. In this example, the heater temperature $T_{h}=385^{\circ} \mathrm{C}$ produces a temperature gradient $\Delta T=$ $38^{\circ} \mathrm{C}$, evaluated as the difference between the average temperatures in the waveguide silicon cores $\left(165^{\circ} \mathrm{C}\right.$ and $127^{\circ} \mathrm{C}$, respectively).

To measure the TDC performance we realized a set of racetrack resonators (RRs) in all-pass filter configuration such as that shown in Fig. 1(b). The $K$ values were extracted using the equation given in [11] from RRs with a radius of $20 \mu \mathrm{m}$ and a round-trip geometric length $L_{r}=270.5 \mu \mathrm{m}$. Figure 2 shows the wavelength domain response of one of the fabricated RRs when the $K$ value of the TDC ( $\left.g=300 \mathrm{~nm}, L_{c}=74 \mu \mathrm{m}\right)$ is thermally tuned. The average round-trip loss is around $0.26 \mathrm{~dB} /$ turn, which corresponds to a field round-trip loss parameter $\gamma=0.97$. When no power is dissipated over the heater $\left(P_{D}=0\right.$, blue trace $\left.T_{1}\right), K=0.7$ and the resonator is highly overcoupled $\left(K \gg 1-\gamma^{2}\right)$. When the heater temperature increases, the power coupled into the RR decreases because of the thermally induced $\Delta n_{t h}$. As a result, the notch depth progressively increases until the critical coupling condition is reached $\left(P_{D}=30.4 \mathrm{~mW}\right.$, red trace $T_{2}$ ). Here $K=0.06 \approx 1-\gamma^{2}$, the notch depth is maximum $(\approx 33.3 \mathrm{~dB})$, and the $Q$ factor is in the order of 40,000 (see Fig. 2 inset). A further increase of $P_{D}$ undercouples the resonator $\left(P_{D}=33.9 \mathrm{~mW}\right.$, green trace $T_{3}$ ), so that the notch depth monotonically decreases as $K$ reduces.

To maximize the TDC efficiency, systematic 2D thermo-optical simulations were performed assuming a thermal conductivity of $163,1.38$, and $1 \mathrm{~W} /(\mathrm{m} \cdot \mathrm{K})$ for $\mathrm{Si}, \mathrm{SiO}_{2} \mathrm{BOX}$, and oxide overcladding layers, respectively $[12,13]$. The temperature dependence of the Si thermooptic coefficient was also considered [14]. As a boundary condition, we fixed room temperature $\left(25^{\circ} \mathrm{C}\right)$ at the boundaries of the $40 \mu \mathrm{m} \times 83 \mu \mathrm{m}$ computational window. From the simulated cross-sectional thermal profile [see Fig. 1(c)], we calculated the effective index difference $\Delta n_{t h}$ and the resulting phase mismatch term $\Delta \beta$ induced by the transverse temperature gradient $\Delta T$.

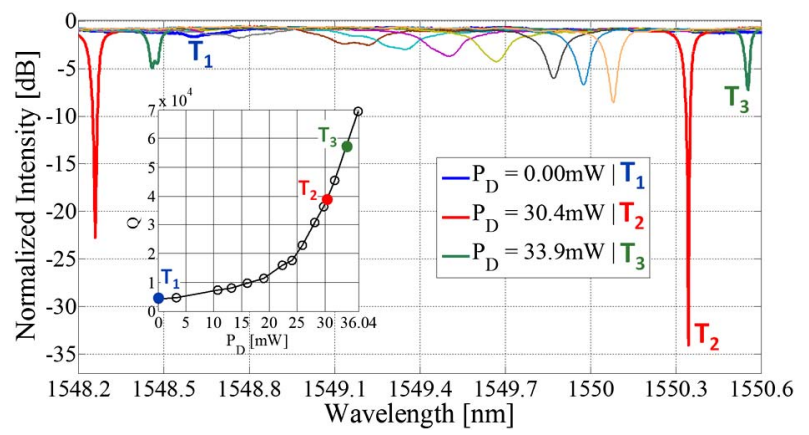

Fig. 2. (Color online) Wavelength domain response of an RR all-pass filter for increasing power $P_{D}$ dissipated in the TDC: overcoupling $\left(P_{D}=0, K=0.7\right.$, blue curve $\left.T_{1}\right)$, critical coupling $\left(P_{D}=30.4, K=0.06\right.$, red curve $\left.T_{2}\right)$, and undercoupling condition $\left(P_{D}=33.9, K=0.022\right.$, green curve $\left.T_{3}\right)$.
Figure 3(a) shows the simulated performance of three synchronous $(\Delta n=0)$ TDCs with different gaps $g$ as a function of the heater lateral shift $d$. The value of $\Delta n_{t h}$ is normalized to $\Delta n_{t h 0}$, the effective index difference necessary to switch off the coupler $(K=0)$. The couplers' lengths $L_{c}$ were chosen to have $K=1$ when $P_{D}=0$. Independently of the dissipated power [two examples are shown in Fig. $3\left(\right.$ a) with $P_{D}=16$ and $29 \mathrm{~mW}$ ], a maximum tuning efficiency is reached for $d$ of about $0.6 \mu \mathrm{m}$. The experimental results of Fig. 3(b) confirm simulations, showing that the highest tuning efficiency is provided by the heater with the larger lateral shift, enabling us to reduce $K$ down to 0.52 with $P_{D}=29 \mathrm{~mW}$. The same power dissipation leads to $K=0.57$ and 0.68 for $d=0$ and $-0.3 \mu \mathrm{m}$, respectively.

Figure 3(a) also shows that the tuning efficiency is almost independent of the coupler gap $g$. We verified it experimentally by realizing four synchronous TDCs, two with nominal $K=1$ and $g=200$ or $250 \mathrm{~nm}$, and two with nominal $K=0.5$ and $g=200$ or $300 \mathrm{~nm}$. The heater offset is fixed to $d=0.6 \mu \mathrm{m}$. In Fig. 4 , we can see that TDCs with the same nominal $K$, but different $g$, have almost the same efficiency, the small difference being addressable to fabrication tolerances [9]. Moreover we can notice device efficiency independence on the solution order of Eq. (1). The TDCs with $K=0.5$ were in fact designed with $S L_{c}=\pi / 4$ (black trace) and $S L_{c}=3 \pi / 4$ (gray trace).
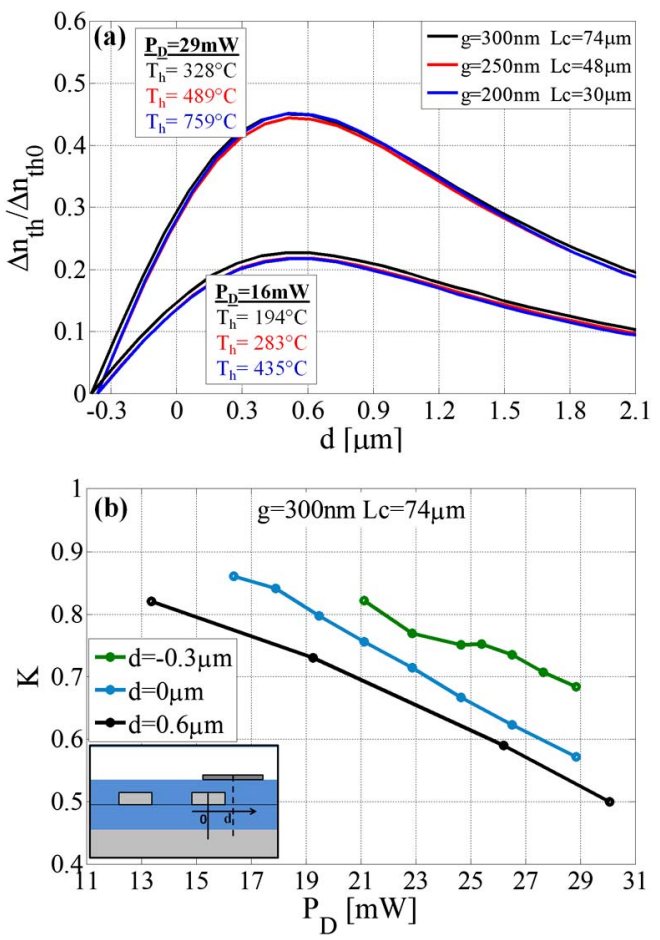

Fig. 3. (Color online) (a) Normalized thermally induced effective index difference versus $d$ for three TDCs with $g=200 \mathrm{~nm}$ (blue curve), $250 \mathrm{~nm}$ (red curve), and $300 \mathrm{~nm}$ (black curve). The coupling length $L_{c}$ is chosen to have $K=1$ for $P_{D}=0$. Heater temperatures $T_{h}$ refer to the case $d=0.6 \mu \mathrm{m}$. (b) Measured $K$ of three synchronous TDCs with $g=300 \mathrm{~nm}$ and $L_{c}=74 \mu \mathrm{m}$ for $d=-0.3 \mu \mathrm{m}$ (green circles), $0 \mu \mathrm{m}$ (blue circles), and $0.6 \mu \mathrm{m}$ (black circles). 


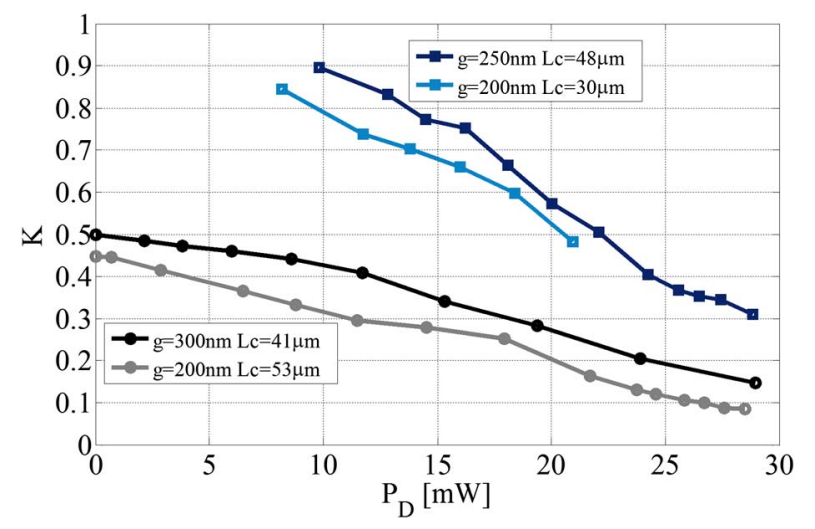

Fig. 4. (Color online) Measured $K$ versus $P_{D}$ of four synchronous TDCs with nominal $K=1$ (squares, $g=200$ and $250 \mathrm{~nm}$ ) and $K=0.5$ (circles, $g=200$ and $300 \mathrm{~nm}$ ). The heater offset is $d=0.6 \mu \mathrm{m}$.

It should be noted that a lower bound exists for $L_{c}$, set by the fuse temperature of the heaters. Lower values of $L_{c}$, keeping $K$ constant, can be obtained by decreasing $g$. However, the same $P_{D}$ dissipated over a shorter length results in a higher heater temperature $T_{h}$ [see Fig. 3(a)].

Finally, in Fig. 5, the simulated (solid lines) and measured (circles) $K$ of three synchronous TDCs for several coupling lengths $L_{c}=52 \mu \mathrm{m}$ (blue), $57 \mu \mathrm{m}$ (orange), and $74 \mu \mathrm{m}$ (black) are compared. The gap distance and lateral shift of the heater are $g=300 \mathrm{~nm}$ and $d=0.6 \mu \mathrm{m}$, respectively. Experimental results agree well with the model, predicting that the maximum $K$ can be reduced by about three times with $P_{D}=35 \mathrm{~mW}$ and that more than $50 \mathrm{~mW}$ are required to bring $K$ below 0.05 . Such a low efficiency is expected from Eq. (1), stating that for synchronous TDCs $(\Delta n=0)$, the derivative of $K$ versus $\Delta \beta$ (that is, versus $P_{D}$ ) vanishes at a small $P_{D}$. The tuning efficiency can be increased by designing asynchronous TDCs $(\Delta n \neq 0)$ in order to steepen the $K-P_{D}$ characteristic. Diamonds and the gray dashed-dotted trace in Fig. $\underline{5}$

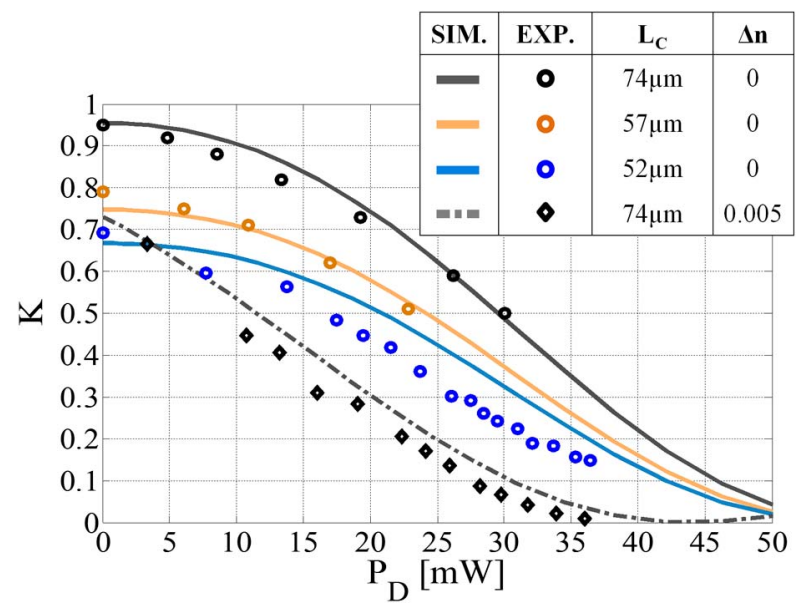

Fig. 5. (Color online) Measured (circles) and simulated (solid curves) $K$ versus $P_{D}$ of three synchronous TDCs with coupling length $L_{c}=52 \mu \mathrm{m}$ (blue), $57 \mu \mathrm{m}$ (orange), and $74 \mu \mathrm{m}$ (black). Diamonds and the dashed-dotted line show the performance of an asynchronous TDC $(\Delta n=0.006)$ with $L_{c}=74 \mu \mathrm{m}$. All the devices have $g=300 \mathrm{~nm}$ and $d=0.6 \mu \mathrm{m}$. show the measured and simulated performance of an asynchronous TDC with the same $g, d$, and $L_{c}$ parameters of that indicated by the black curve. The only difference is that one of the two coupled waveguides is narrowed to $w=477 \mathrm{~nm}$ in order to have an initial $\Delta n=0.005$. Due to this intrinsic phase mismatch, the tuning curve decreases linearly at small $P_{D}$ with a $K-P_{D}$ slope of $0.021 \mathrm{~mW}^{-1}$. Less than $32 \mathrm{~mW}$ are required to bring $K$ from 0.7 to 0.05 , and typical thermo-optic actuator time responses are in the order of $10 \mu \mathrm{s}$ [9].

In conclusion, we have presented a silicon photonic TDC driven by a transverse temperature gradient induced by a laterally shifted integrated heater. Through a systematic numerical and experimental investigation, we demonstrated that the tuning efficiency is almost independent of the waveguide gap distance and we found the optimum heater position to minimize power consumption. Tuning efficiency can be increased by designing TDCs with an intrinsic phase mismatch. A tunable split ratio from 0.7 to 0.01 requiring $36 \mathrm{~mW}$ of power dissipation was achieved in a $74 \mu \mathrm{m}$ long device. Due to its small footprint, the proposed TDC is a valuable alternative to conventional MZI-based schemes in PICs where extremely compact tunable couplers are required, such as in RRs with large free spectral ranges.

Part of this work has been funded by the Italian PRIN 2009 project Shared Access Platform to Photonic Integrated Resources (SAPPHIRE). The authors thank the James Watt Nanofabrication Centre (JWNC) staff at Glasgow University for the fabrication of the devices.

\section{References}

1. H. L. R. Lira, C. B. Poitras, and M. Lipson, Opt. Express 19, 20115 (2011).

2. P. Orlandi, C. Ferrari, M. J. Strain, A. Canciamilla, F. Morichetti, M. Sorel, P. Bassi, and A. Melloni, Opt. Lett. 37, 3669 (2012).

3. M. S. Rasras, K.-Y. Tu, D. M. Gill, Y.-K. Chen, A. E. White, S. S. Patel, A. Pomerene, D. Carothers, J. Beattie, M. Beals, J. Michel, and L. C. Kimerling, J. Lightwave Technol. 27, 2105 (2009).

4. E. J. Norberg, R. S. Guzzon, S. C. Nicholes, J. S. Parker, and L. A. Coldren, Photon. Technol. Lett. 22, 109 (2010).

5. L. Luo, S. Ibrahim, A. Nitkowski, Z. Ding, C. B. Poitras, S. J. Ben Yoo, and M. Lipson, Opt. Express 18, 23079 (2010).

6. H. Kogelnik and R. V. Schmidt, IEEE J. Quantum Electron. 12, 396 (1976).

7. Y. Silberberg, P. Perlmutter, and J. E. Baran, Appl. Phys. Lett. 51, 1230 (1987).

8. J. Leuthold and C. H. Joyner, J. Lightwave Technol. 19, 700 (2001).

9. A. Melloni, A. Canciamilla, C. Ferrari, F. Morichetti, L. O'Faolain, T. F. Krauss, R. De La Rue, A. Samarelli, and M. Sorel, IEEE Photonics J. 2, 181 (2010).

10. A. Yariv and P. Yeh, Photonics: Optical Electronics in Modern Communications (Oxford University, 2007), p. 614.

11. O. Schwelb, J. Lightwave Technol. 22, 1380 (2004).

12. V. M. N. Passaro, F. Magno, and A. V. Tsarev, Opt. Express 13, 3429 (2005).

13. A. H. Atabaki, E. S. Hosseini, A. A. Eftekhar, S. Yegnanarayanan, and A. Adibi, Opt. Express 18, 18312 (2010).

14. G. Cocorullo, F. G. Della Corte, and I. Rendina, Appl. Phys. Lett. 74, 3338 (1999). 